\title{
THE $K$-THEORY OF A CLASS OF HOMOGENEOUS SPACES
}

\author{
BY \\ BRUNO HARRIS $\left({ }^{1}\right)$
}

1. Introduction. We calculate the groups $K^{*}(G / U)$ modulo torsion $\left(K^{*}\right.$ denoting the Grothendieck-Atiyah-Hirzebruch ring based on complex vector bundles) for homogeneous spaces $G / U$ satisfying the following conditions: $G$ is a compact, connected, simply-connected simple Lie group, $U$ is a closed subgroup which is totally nonhomologous to zero in $G$ for rational coefficients, and $\tau$ is an automorphism of period 2 of $G$ with $U$ as fixed point set. Since $G$ is simple, it must be $\mathrm{SU}(n)$, Spin $(2 n)$, or $E_{6}$ (see $\left.\S 3\right)$ and the simplest example of such a "symmetric space" $G / U$ is $S^{2 n-1}=\operatorname{Spin}(2 n) / \operatorname{Spin}(2 n-1)$.

We use Atiyah's result [1] that $K^{*}(G) \bmod$ torsion is an exterior algebra $\bigwedge\left(\left[\rho_{1}\right], \ldots,\left[\rho_{l}\right]\right)$ where the $\rho_{i}$ are the basic irreducible representations of $G$. Hodgkin's theorem that $K^{*}(G)$ is actually torsion free will be used only in a few places to sharpen the results (see Hodgkin [10], or a forthcoming paper of Araki).

For any space $X$ let $K_{f}^{*}(X)$ denote $K^{*}(X)$ modulo torsion. $G$ and $U$ will be assumed to satisfy the conditions in the first paragraph unless otherwise stated. The first theorem describes $K_{f}^{*}(G / U)$ and the homomorphisms:

$$
K_{f}^{*}(G / U) \stackrel{p^{*}}{\longrightarrow} K_{f}^{*}(G) \stackrel{q^{*}}{\longrightarrow} K_{f}^{*}(G / U)
$$

where $p: G \rightarrow G / U$ is the natural projection, and $q: G / U \rightarrow G$ is given by $q(g U)$ $=g \tau(g)^{-1}(\tau$ is the automorphism of $G)$. The second theorem uses the relation of $G / U$ to a certain Jordan algebra to show that $K_{f}^{*}(G / U)$ as a ring with operations is generated by one element defined by means of the Jordan algebra (e.g., a halfspin representation for $\left.S^{2 n-1}\right)$. The third theorem relates $K_{f}^{*}(G / U)$ to $K_{f}^{*}(\Omega(L / G))$ where $L / G$ is again a homogeneous space and $\Omega$ denotes its loop space. The fourth theorem applies these results to fiber bundles with $G / U$ as fiber.

We now state these theorems:

THEOREM 1. (a) $K_{f}^{*}(G / U)$ is an exterior aigebra $\wedge\left(x_{1}, \ldots, x_{r}\right)$ and $p^{*}: K_{f}^{*}(G / U)$ $\rightarrow K_{f}^{*}(G)$ is injective, $q^{*}: K_{f}^{*}(G) \rightarrow K_{f}^{*}(G / U)$ is surjective.

(b) $K_{f}^{*}(G)=\bigwedge\left(\left[\rho_{1}\right], \ldots,\left[\rho_{r}\right], \tau^{*}\left[\rho_{1}\right], \ldots, \tau^{*}\left[\rho_{r}\right],\left[\rho_{r+1}\right], \ldots,\left[\rho_{l}\right]\right)$ where $\tau^{*}\left[\rho_{i}\right]=$ $\left[\rho_{l}\right]$ for $i>r$, and $K_{f}^{*}(G / U)=\bigwedge\left(q^{*}\left[\rho_{1}\right], \ldots, q^{*}\left[\rho_{\mathrm{r}}\right]\right)$ while $q^{*}\left[\rho_{i}\right]=0$ for $i>r$.

(c) $p^{*} K_{f}^{*}(G / U)=\bigwedge\left(\left[\rho_{1}\right]-\tau^{*}\left[\rho_{1}\right], \ldots,\left[\rho_{r}\right]-\tau^{*}\left[\rho_{r}\right]\right) \subset K_{f}^{*}(G)$.

Received by the editors December 22, 1966.

(1) Work supported in part by NSF Grant GP-3446. 
The proof of Theorem 1, with some similar results for more general homogeneous spaces, is given in $\S 2$. The proof is very short and does not require the classification theory.

The next theorem gives a more precise description of $K_{f}^{*}(G / U)$, including opera tions, for the class of symmetric spaces described in the first paragraph. With each such space we associate a central simple formally-real Jordan algebra $J$ (see [5] and its references) such that $U$ is the automorphism group of $J$, and $G$ is the compact simply-connected form of the group $N(J)$ of linear transformations of $J$ preserving the norm form (a kind of determinant) of $J$.

THEOREM 2. Let $R$ be a complex irreducible representation of the Jordan algebra $J \otimes_{R} \boldsymbol{C}$, which is a special representation if $J$ is a special Jordan algebra. Then $R$ determines a complex irreducible representation of $G$ and a corresponding element $[\rho]$ of $K_{f}^{-1}(G)$, and

$$
K_{f}^{*}(G / U)=\left(q^{*}[\rho], q^{*}\left[\lambda^{2} \rho\right], \ldots, q^{*}\left[\lambda^{r} \rho\right]\right)
$$

where $q: G / U \rightarrow G$ is the imbedding, the $\lambda^{i}$ are exterior powers and $r=$ rank of $G-$ rank of $U$.

The proof of Theorem 2, in $\S 3$, makes use of the classification. However, it is short and illustrates the concepts involved.

If $G / U$ is a symmetric space as before, or under more general circumstances, then one can find Lie groups $L$ containing $G$ and Bott maps $B: G / U \rightarrow \Omega(L / G)$, $\Omega$ denoting the loop space. The composition of $B$ and the natural map $\omega: \Omega(L / G)$ $\rightarrow G$ is just $q: G / U \rightarrow G$ and we obtain

THEOREM 3. $K_{f}^{*}(\Omega(L / G)) \approx K_{f}^{*}(G / U) \oplus \operatorname{Ker} B^{*}$.

Finally, we make an application to fiber bundles. Let $G, U$ be as in Theorems 1 and 2, and let $\rho$ be the representation of $G$ described in Theorem 2. Let $P \rightarrow P / G$ be a principal $G$-bundle and $G / U \rightarrow P / U \rightarrow P / G$ the associated $G / U$-bundle. Let ( $\rho)$ and $\tau^{*}(\rho)=(\rho \circ \tau)$ be the corresponding elements of $K^{\circ}(P / G)$. We then have:

Theorem 4. Let $(\rho)-\tau^{*}(\rho)=0$ in $K^{0}(P / G)$. Then $K_{f}^{*}(P / U) \rightarrow K_{f}^{*}(G / U)$ is surjective. If further $K^{*}(G / U)$ is torsion-free then $K^{*}(P / U) \approx K^{*}(P / G) \otimes K^{*}(G / U)$ as algebras.

The results stated for symmetric spaces actually hold more generally but in more complicated form, as shown in the following sections.

2. By $[1], K_{f}^{*}(G)=\bigwedge\left(\left[\rho_{1}\right], \ldots,\left[\rho_{l}\right]\right)$ where $\rho_{1}, \ldots, \rho_{l}$ are the basic irreducible representations $\rho_{i}: G \rightarrow U\left(N_{i}\right)$ and $\left[\rho_{i}\right]$ are the corresponding elements in $K_{f}^{-1}(G)$.

We recall that $\operatorname{ch}: K_{f}^{*}(G) \rightarrow H^{*}(G ; \boldsymbol{Q})$ is injective and identifies $K^{*}(G) \otimes \boldsymbol{Q}$ with $H^{*}(G ; Q)$. Elements $x$ of $K_{f}^{*}(G)$ will be called primitive if $\operatorname{ch} x$ is primitive in the Hopf algebra $H^{*}(G ; \boldsymbol{Q})$. 
It is easy to see that the $\left[\rho_{i}\right]$ are a basis for the primitive elements $P K_{f}^{*}(G)$ in $K_{f}^{*}(G)$ (for, one may look at $K^{-1}(U(N))$ and the element $[R]$ corresponding to the standard identity representation; $[R]$ is primitive since ch $[R]$ is the suspension of an element in $H^{*}\left(B_{U(N)}\right)$.

Let $p: G \rightarrow G / U, q: G / U \rightarrow G$ be given by $p(g)=g U, q(g U)=g \tau(g)^{-1}$. Thus $q \circ p(g)=g \tau(g)^{-1}$. An easy calculation (see [4]) shows that if $y \in H^{*}(G ; Q)$ is primitive then $p^{*} q^{*}(y)=y-\tau^{*} y$. Thus

$$
p^{*} q^{*}\left[\rho_{i}\right]=\left[\rho_{i}\right]-\tau^{*}\left[\rho_{i}\right]=\left[\rho_{i}\right]-\left[\tau\left(\rho_{i}\right)\right]
$$

The action of $\tau$ on the $\rho_{i}$, defined by $\tau\left(\rho_{i}\right)=\rho_{i} \circ \tau$, is easily described: the $\rho_{i}$ are in 1-1 correspondence with the vertices of the Dynkin diagram of $G$, and $\tau$ (modulo inner automorphisms) is determined by a permutation of these vertices which is an automorphism of the diagram. Thus $\tau$ permutes the $\rho_{i}$, and $\tau^{2}$ gives the identity permutation. Hence we may relabel the $\rho_{i}$ as $\rho_{1}, \ldots, \rho_{r}, \tau \rho_{1}, \ldots, \tau \rho_{r}, \rho_{1}^{\prime} \ldots, \rho_{s}^{\prime}$ where $\tau \rho_{i}^{\prime}=\rho_{i}^{\prime}$, and $2 r+s=l$. Thus $K_{f}^{*}(G)=\bigwedge^{\prime \prime} \otimes \tau^{*} \bigwedge^{\prime \prime} \otimes \wedge^{\prime}, \bigwedge^{\prime \prime}=\bigwedge\left(\rho_{1}, \ldots, \rho_{r}\right)$, $\bigwedge^{\prime}=\bigwedge\left(\rho_{1}^{\prime}, \ldots, \rho_{s}^{\prime}\right)$. The image of $p^{*} q^{*}$ is $\wedge\left(\rho_{1}-\tau \rho_{1}, \ldots, \rho_{r}-\tau \rho_{r}\right)$, an abelian group direct summand of $K_{f}^{*}(G)$. We note that $\tau^{*}\left[\rho_{i}^{\prime}\right]=\left[\rho_{i}^{\prime}\right]$ implies $q^{*}\left[\rho_{i}^{\prime}\right]=0$ : for $\tau$ operates on $G / U$ so that $q \tau=\tau q$ and $\tau q(g K)=q(g K)^{-1}=u(q(g K))$ where $u(g)=g^{-1}$ in $G$; thus $q^{*}\left[\rho_{i}^{\prime}\right]=q^{*} \tau^{*}\left[\rho_{i}^{\prime}\right]=q^{*} u^{*}\left[\rho_{i}^{\prime}\right]=-q^{*}\left[\rho_{i}^{\prime}\right]$ since $u^{*}(x)=-x$ for any primitive $x$.

Thus $q^{*}=0$ on $\bigwedge^{\prime}, q^{*} \bigwedge^{\prime \prime}=q^{*} \tau^{*} \bigwedge^{\prime \prime}=\operatorname{Im} q^{*}, p^{*} q^{*}$ maps $\bigwedge^{\prime \prime}$ isomorphically onto a direct summand, and $K_{f}^{*}(G / U)=\operatorname{Im} q^{*} \oplus \operatorname{Ker} p^{*}$. However, we assumed $U$ nonhomologous to zero in $G$ (over $Q$ ) so $p^{*}$ is $1-1$, and $K_{f}^{*}(G / U)=\operatorname{Im} q^{*}=q^{*} \wedge^{\prime \prime}$ $\approx \bigwedge^{\prime \prime}$. Theorem 1 is now proved.

The argument used to prove Theorem 1 also applies in more general situations: let $U$ be a closed subgroup of $G$, with $G$ compact, connected, simply connected, and let $\rho_{1}, \rho_{1}^{\prime}, \rho_{2}, \rho_{2}^{\prime} \cdots$ be representations of $G$ such that $\rho_{i}=\rho_{i}^{\prime}$ on $U$. Then if $\rho_{i}, \rho_{i}^{\prime}: G \rightarrow U\left(N_{i}\right)$, we can define maps $q_{i}: G / U \rightarrow U\left(N_{i}\right)$ by $q_{i}(g U)=\rho_{i}(g) \rho_{i}^{\prime}(g)^{-1}$ and corresponding elements $\left[q_{i}\right] \in K^{-1}(G / U),\left[\rho_{i}\right] \in K^{-1}(G)$. An example of this occurs when $\rho_{i}$ is a representation of $G, \tau$ an automorphism of $G$ which is the identity on $U$, and $\rho_{i}^{\prime}=\rho_{i} \circ \tau$ : then $q_{i}=\rho_{i} \circ q$ where $q: G / U \rightarrow G$ is as before.

If $p: G \rightarrow G / U$ is the projection, then $p^{*}\left[q_{i}\right]=\left[\rho_{i}\right]-\left[\rho_{i}^{\prime}\right] \in K^{*}(G)$. With this notation, we now have:

Proposition 2.1. Let $M$ be the submodule of $K_{f}^{-1}(G)$ generated by the elements $\left[\rho_{i}\right]-\left[\rho_{i}^{\prime}\right]$. Then $K_{f}^{*}(G / U)$ contains a subalgebra $A$ isomorphic under $p^{*}$ to $\wedge(M)$.

Proof. Pick a basis $\left\{m_{j}\right\}$ of $M$, let $a_{j} \in K_{f}^{-1}(G / U), p^{*} a_{j}=m_{j} . A$ is generated by the $a_{j}$.

Proposition 2.2. Let $\tau$ be an outer automorphism of $G$ of prime order (modulo inner automorphisms) which is the identity on $U$. Then

1. $K_{f}^{*}(G)=\bigwedge(M) \otimes \bigwedge(N)$ where $M, N \subset K_{f}^{-1}(G), \tau$ is the identity on $N$ and $M=Z[\tau] \otimes M^{\prime}$ is a free module over the group algebra $Z[\tau]$ of $\tau$. 
2. $K_{f}^{*}(G / U)=A \otimes B$ where

$$
p^{*}: A \stackrel{\approx}{\longrightarrow}\left((\tau-1) Z(\tau) \otimes M^{\prime}\right)
$$

and $B$ is an ideal.

To prove Proposition 2.2, we pick a basic representation $\rho_{i}$ of $G$ from each orbit of $\tau$ (acting on basic representations) and define an element $x_{i, j}$ of $K^{-1}(G / U)$ by $x_{i, j}=q^{*}\left[\tau^{j} \rho_{i}\right]$ for $1 \leqq j \leqq($ order of $\tau)-1$. Then $p^{*} x_{i j}=\left[(\tau-1) \tau^{j} \rho_{i}\right]$. The $x_{i, j}$ generate the exterior algebra $A$, while $B$ is the kernel of $p^{*}$ followed by projection on $p^{*}(A)$.

An example for Proposition 2.1 is the Stiefel manifold SU $(n+k) / \mathrm{SU}(n)$ : more generally,

Proposition 2.3. Let $G \supset U$ both be compact connected and simply-connected, and let the map of representation rings $R(G) \rightarrow R(U)$ be surjective. Then $K^{*}(G / U)$ is torsion free and is an exterior algebra generated by elements $\left[q_{i}\right]$ constructed from pairs $\left(\rho_{i}, \rho_{i}^{\prime}\right)$ of representations of $G$ which coincide on $U$.

Proof. By Hodgkin's theorem $K^{*}(G)$ and $K^{*}(U)$ are both torsion free. The relation between $R(G)$ and $K^{-1}(G)$ is the following: if $\bar{R}(G)$ is the kernel of the rank homomorphism then $\bar{R}(G) / \bar{R}(G)^{2}$ is isomorphic to $P K^{-1}(G)$ under the sequence of homomorphisms:

$$
\bar{R}(G) \stackrel{\alpha}{\longrightarrow} \tilde{K}^{0}\left(B_{G}\right) \longrightarrow \tilde{K}^{0}(S(G)) \stackrel{\delta^{-1}}{\longrightarrow} K^{-1}(G)
$$

using the standard map of the suspension $S(G)$ into $B_{G}$. The same things hold for $U$. Hence if $i^{*}: R(G) \rightarrow R(U)$ is surjective then so is $K^{*}(G) \rightarrow K^{*}(U)$. It follows that $K^{*}(G / U) \rightarrow K^{*}(G)$ is injective and has as image the exterior algebra on $M=\operatorname{Ker} i^{*}: P K^{*}(G) \rightarrow P K^{*}(U)$. Thus the proposition reduces to proving that if $m \in M$ then we can find representations $\rho, \rho^{\prime}$ of $G$ which coincide on $U$ such that $m=[\rho]-\left[\rho^{\prime}\right]$.

Let $m=\sum n_{j}\left[\rho_{j}\right], \rho_{j}$ being basic, and let

$$
\mu=\sum\left\{n_{j} \rho_{j} \mid n_{j}>0\right\}, \quad \mu^{\prime}=\sum\left\{n_{j} \rho_{j} \mid n_{j}<0\right\} .
$$

By adding to $\mu$ or $\mu^{\prime}$ suitably many copies of the trivial one-dimensional representation we may assume $\mu-\mu^{\prime} \in \bar{R}(G)$. Also, since $i^{*} m=0$, we have $i^{*} \mu-i^{*} \mu^{\prime}$ $\in \bar{R}(U)^{2}$. Since $i^{*} R(G)=R(U)$, we also have $i^{*} \bar{R}(G)^{2}=\bar{R}(U)^{2}$, so we can find $\gamma \in \bar{R}(G)^{2}$ with $i^{*}(\gamma)=i^{*}\left(\mu-\mu^{\prime}\right)$. Writing $\gamma=\gamma_{2}-\gamma_{1}$ where the $\gamma_{i}$ are representations of $G$ and letting $\rho=\mu+\gamma_{1}, \rho^{\prime}=\mu^{\prime}+\gamma_{2}$ we have $m=[\rho]-\left[\rho^{\prime}\right]$ and $i^{*} \rho=i^{*} \rho^{\prime}$, as required.

3. We shall prove Theorem 2 by looking at the classification of the symmetric pairs $(G, U)$ such that $G$ is simple, compact, and simply-connected, and $U$ is totally nonhomologous to zero in $G$ with rational coefficients. We will find in each case a central simple Jordan algebra $J$ over $\boldsymbol{R}$ such that 
(a) the Lie algebra $D$ of derivations of $J$ is the Lie algebra of $U$ and consists of all linear transformations of the form $\sum_{i}\left[R a_{i}, R b_{i}\right]$ where $R a_{i}(x)=x a_{i}$ all $x \in J$, and $a_{i}, b_{i}$ are elements of $J$ of trace 0 ,

(b) the Lie algebra $\mathscr{S S}_{0}$ of linear transformations of $J$ of the form $R_{a}+d$, a of trace zero in $J, d \in D$, is a noncompact real form of the Lie algebra $\mathbb{B}$ of $G$ : i.e., (S) $=\left\{i R_{a}+d\right\} \subset \mathcal{S S}_{0} \otimes_{R} C$. (S) is also the Lie algebra of norm-preserving linear transformations in $J$.

Any complex irreducible representation of $J \otimes_{R} C$ then determines a complex irreducible representation of $B$. We shall now list these:

(i) $G=\mathrm{SU}(2 n+1), U=\mathrm{SO}(2 n+1)$. Here $J$ is the Jordan algebra of all real symmetric matrices of $2 n+1$ rows. $J \otimes_{R} C$ is defined in the same way but with complex matrices, and the representation is the obvious one on $C^{2 n+1}$. The representation $\rho$ of $\mathrm{SU}(2 n+1)$ is the standard one, and it is known that the basic representations can be taken as $\rho_{i}=\lambda^{i} \rho, 1 \leqq i \leqq 2 n$.

(ii) $G=\mathrm{SU}(2 n), U=\operatorname{Sp}(n)$. Here $J$ is the Jordan algebra of all $n$-rowed quaternion hermitian matrices, $J \otimes_{R} C$ consists of all $2 n$-rowed symplectic-symmetric matrices with complex coefficients. The representation of $G$ obtained is the standard one on $C^{2 n}$, and the concluding remark of case (i) again applies.

(iii) $G=\operatorname{Spin} 2 n, U=\operatorname{Spin} 2 n-1 . J$ is the Jordan algebra of the vector space $\boldsymbol{R}^{2 n-1}$ with the Euclidean inner product. The universal associative enveloping algebra $U_{s}(J)$ is the Clifford algebra $C_{2 n-1}=C\left(\boldsymbol{R}^{2 n-1}\right)$ and the complex irreducible representation of Spin $2 n$ is a half-spin representation.

(iv) $G=E_{6}, U=F_{4}$. Here $J$ is the Jordan algebra of 3-rowed Hermitian matrices with Cayley number coefficients: its dimension over $\boldsymbol{R}$ is 27. The representation $\rho$ of $G$ obtained is the obvious representation on $J \otimes_{R} C$. Here the "rank" $r$ is 2 , and we will show that $\rho, \lambda^{2} \rho, \tau^{*} \rho$ and $\tau^{*} \lambda^{2} \rho$ are four of the basic irreducible representations of $E_{6}$, the remaining two representations being fixed under $\tau$. We look at the Dynkin diagram of $E_{6}$, with dimensions written next to the vertices:

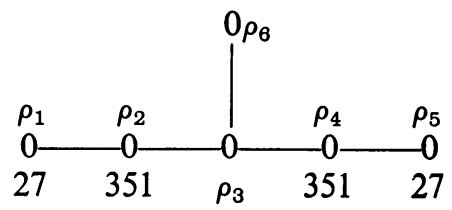

$\rho=\rho_{1}$ corresponds to the leftmost vertex. A simple observation of Dynkin's ([3, p. 347]) is that the highest weight vector occuring in $\lambda^{2} \rho_{1}$ is the highest weight vector of the next representation, $\rho_{2}$. Thus $\rho_{2}$ occurs in the decomposition of $\lambda^{2} \rho_{1}$ into irreducible parts; however, the dimensions of $\rho_{2}$ and of $\lambda^{2} \rho_{1}$ coincide, so $\rho_{2}=\lambda^{2} \rho_{1}$.

$\tau$ being a symmetry of the Dynkin diagram, it is clear that $\tau^{*} \rho_{1}=\rho_{5}, \tau^{*} \rho_{2}=\rho_{4}$, $\tau^{*} \rho_{3}=\rho_{3}, \tau^{*} \rho_{6}=\rho_{6}\left(\rho_{6}\right.$ is the adjoint representation).

This concludes the proof of Theorem 2 . 
It seems likely that a proof of Theorem 2 without use of the classification could be carried out by considering the Dynkin diagram of 55, identifying the representation $\rho$ as associated with an end vertex (e.g., $\rho_{1}$ for $E_{6}$ ), and, finally, discussing the exterior powers $\lambda^{i} \rho$ and the automorphism $\tau$ of the Dynkin diagram.

The classification used in the proof of Theorem 2 can be used to show that the map of representation rings $R(G) \rightarrow R(U)$ is surjective: this is easy to see if $U$ is a classical group, whereas if $G=E_{6}, U=F_{4}$ then one can show that the representation ring of $F_{4}$ is generated by the 26 dimensional representation on $J$ and the adjoint representation, and both of these are in the image of $R\left(E_{6}\right) \rightarrow R\left(F_{4}\right)$. Since $(\operatorname{Sp} n)$, (Spin $2 n-1)$, and $F_{4}$ are simply-connected groups their $K^{*}$ groups are torsion free by Hodgkin's theorem, so that by Proposition $2.3, K^{*}(G / U)$ is torsion free in cases (ii), (iii), and (iv) of the classification; similarly one can see that $K^{*}(\mathrm{SU}(2 n+1) / \mathrm{SO}(2 n+1))$ has no $p$-torsion for $p$ odd, but it probably has 2-torsion.

Finally, one can show by a general argument without case considerations that $K^{*}(G / U)$ in all cases has no $p$-torsion for $p$ odd (using again Hodgkin's theorem): one starts with the result from [11] or [6] that the composite map $p \circ q$ :

$$
G / U \stackrel{q}{\longrightarrow} G \stackrel{p}{\longrightarrow} G / U
$$

induces an automorphism in cohomology with coefficients $Z_{p}$, or, equivalently, an automorphism on $H^{*}\left(G / U ; Z\left(\frac{1}{2}\right)\right), Z\left(\frac{1}{2}\right)$ denoting the subring of $Q$ generated by $\frac{1}{2}$. By the spectral sequence leading from $E_{2}=H^{*}$ to $E_{\infty}=\operatorname{Gr}\left(K^{*} \otimes Z\left(\frac{1}{2}\right)\right)$ one sees that $p^{*}$ is $1-1$ (and $q^{*}$ is onto) on $K^{*}(G / U) \otimes Z\left(\frac{1}{2}\right)$, and since $K^{*}(G) \otimes Z\left(\frac{1}{2}\right)$ is torsion free, so is $K^{*}(G / U) \otimes Z\left(\frac{1}{2}\right)$.

4. Bott [8], [9] has described maps $B: G / U \rightarrow \Omega(L / G)$ if $G / U$ and $L / G$ are suitably related symmetric spaces. For our purposes we will need only the more elementary considerations of [4], which we repeat with a slight change in notation.

We will assume the following data: $U \subset G \subset L$ are compact Lie groups, with $G$ and $L$ connected. $\tau$ is an automorphism of $G$, and $v(t), t \in \boldsymbol{R}$, is a one-parameter subgroup of $L$ satisfying:

(i) $v(1)^{-1} g v(1)=\tau(g), \quad$ all $g \in G$,

(ii) $v(t)^{-1} u v(t)=u$, all $u \in U$.

Define $B: G / U \rightarrow \Omega(L / G)$ by $B(g U)(t)=v(t)^{-1} g v(t) G, 0 \leqq t \leqq 1$. Now consider the fiber bundles

$$
G \stackrel{j}{\longrightarrow} L \stackrel{l}{\longrightarrow} L / G
$$

from general principles we have maps

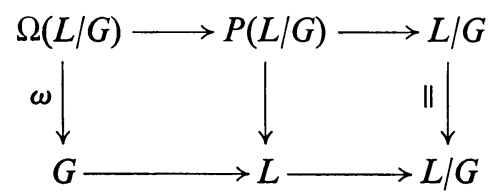


where the first row is the path space fibration on $L / G$. We wish to show that the composition $\omega \circ B: G / U \rightarrow \Omega(L / G) \rightarrow G$ coincides with $q$. It will suffice to construct maps $Q: \mathrm{c}(G / U) \rightarrow L$ (c denotes "cone") $b: s(G / U)=\mathrm{c}(G / U) /(G / U) \rightarrow L / G$ with $b$ the adjoint of $B$, giving a commutative diagram:

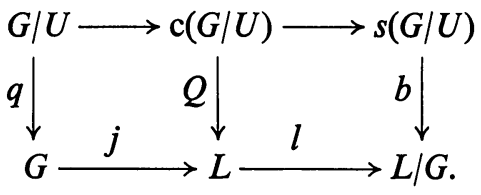

The homotopy class of $b$ determines that of $q$. Similarly we have:



The homotopy class of $b$ determines that of $\omega \circ B$, hence $\omega \circ B$ is homotopic to $q$.

We define

$$
Q(g U, t)=v(t)^{-1} g v(t) \tau(g)^{-1}, \quad b(g U, t)=v(t)^{-1} g v(t) G .
$$

Note that

$$
\begin{aligned}
& Q(g U, 0)=q(g U), \\
& Q(g U, 1)=v(1)^{-1} g v(1) v(1)^{-1} g^{-1} v(1)=e, \\
& Q(e U, t)=e .
\end{aligned}
$$

Thus $Q, b$ satisfy the requirements. We now have: $q^{*}=B^{*} \omega^{*}: K_{f}^{*}(G) \rightarrow K_{f}^{*}(\Omega(L / G))$ $\rightarrow K_{f}^{*}(G / U)$. If $G, U$ are as in Theorem 1 , then $K_{f}^{*}(G)$ contains a subalgebra mapped isomorphically by $q^{*}$, and Theorem 3 follows.

One might ask whether there is an analogue for $K^{*}$ of the following result on the cohomology of the spaces defined above:

Proposition 4.1. Let $p$ be an odd prime. Then $H^{*}\left(\Omega(L / G) ; Z_{p}\right) \approx H^{*}\left(\Omega(L / U) ; Z_{p}\right)$ $\otimes H^{*}\left(G / U ; Z_{p}\right)$ as algebras.

Proof. Consider the principal fiber space

$$
\Omega(L / U) \longrightarrow \Omega(L / G) \stackrel{\pi}{\longrightarrow} G / U
$$

here $\pi$ is the composition of $\omega: \Omega(L / G) \rightarrow G$ and the natural map $G \rightarrow G / U$. We also have the map $B: G / U \rightarrow \Omega(L / G)$. Then $\pi \circ B$ is just the composition $q: G / U$ $\rightarrow G \rightarrow G / U$ which induces an isomorphism in cohomology with coefficients $Z_{p}$ for $p$ odd [6, p. 489]. 
In the case that $G$ and $U$ are associated to a Jordan algebra $J$ as in $\S 3$, a specific group $L$ and a suitable one-parameter subgroup $v(t)$ can be described in terms of $J$, as shown by Kumpel [6] using a construction due to Tits [7].

5. We shall now apply the preceding to fiber bundles.

As always, $G$ will be compact, connected and simply-connected, $U$ a closed connected subgroup. We will say that $K_{f}^{*}(G / U)$ is generated by representations if there are pairs $\left(\rho_{j}, \rho_{j}^{\prime}\right)$ of representations of $G$ that coincide on $U_{2}$ such that the corresponding elements $\left[q_{j}\right] \in K^{-1}(G / U)$ (constructed as in $\S 2$ ) together with elements of $K^{0}(G / U)$ associated with representations of $U$, together generate the algebra $K_{f}^{*}(G / U)$.

If $E \rightarrow E / G$ is a principal $G$-bundle we denote by $\alpha$ the usual homomorphism $R(G) \rightarrow K^{0}(E / G)$.

Proposition 5.1. Let $E \rightarrow E / G$ be a principal G-bundle over a compact base space and let

$$
G / U \stackrel{i}{\longrightarrow} E / U \stackrel{\pi}{\longrightarrow} E / G
$$

be the associated $G / U$-bundle. Suppose that $K_{f}^{*}(G / U)$ is generated by representations and that for each of the generators $\left[q_{j}\right]$ defined by $\left(\rho_{j}, \rho_{j}^{\prime}\right)$ we have $\alpha\left(\rho_{j}\right)-\alpha\left(\rho_{j}^{\prime}\right)=0$ in $K^{0}(E / G)$. Then $i^{*}: K_{f}^{*}(E / U) \rightarrow K_{f}^{*}(G / U)$ is surjective. If further $K^{*}(G / U)$ is torsion-free then $K^{*}(E / U) \approx K^{*}(E / G) \otimes K^{*}(G / U)$ as left $K^{*}(E / G)$-module.

Proof. Clearly, it suffices to show that $i *$ is surjective, and since the generators of $K_{f}^{*}(G / U)$ obtained from representations of $U$ are in the image of $i^{*}$, we only have to prove the same thing for the $\left[q_{j}\right] \in K_{f}^{-1}(G / U)$. We follow the ideas of [2, p. 121]. Let $B$ be the mapping cylinder of $\pi: E / U \rightarrow E / G$, and $B_{1}=E / U \subset B$. Call the inclusion $B_{1} \subset B$ again $\pi$. Similarly, let $C$ be the mapping cylinder of $\pi_{0}: G / U \rightarrow$ pt. (projection into a single point), and $C_{1}=G / U \subset C$. Call the inclusion $C_{1} \subset C$ again $\pi_{0}$ :

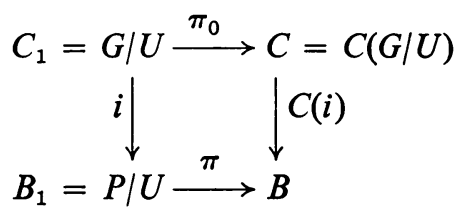

is a commutative diagram, giving rise to:



Let $\rho, \rho^{\prime}$ be representations of $G$ that coincide on $U$. Let $\alpha(\rho), \alpha\left(\rho^{\prime}\right)$ be the associated vector bundles over $B$ (using the homotopy equivalence of $B$ with $P(G)$ ). The 
restrictions to $B_{1}=E / U$ of these bundles are isomorphic, and by the clutching construction define an element $\alpha\left(\rho, \rho^{\prime}\right)$ of $K^{0}\left(B, B_{1}\right)$ which satisfies

$$
j^{*} \alpha\left(\rho, \rho^{\prime}\right)=[\alpha(\rho)]-\left[\alpha\left(\rho^{\prime}\right)\right] \in \tilde{K}^{0}(B)
$$

We will shortly make this clutching function more explicit.

If furthermore the right-hand side of this last equation vanishes, then

$$
\alpha\left(\rho, \rho^{\prime}\right)=\delta \xi \text { for some } \xi \in K^{-1}\left(B_{1}\right) .
$$

Restricting to $C$ and $C_{1}=G / U, i^{*} \xi \in K^{-1}(G / U)$ satisfies

$$
\delta i^{*} \xi=i^{*} \delta \xi=i^{*} \alpha\left(\rho, \rho^{\prime}\right) \in K^{0}(c(G / U), G / U) .
$$

It remains to show that $i^{*} \alpha\left(\rho, \rho^{\prime}\right)$ is determined by the clutching function $q$ on $G / U$, where $q(g U)=\rho(g) \rho^{\prime}(g)^{-1}$ : if we know this, then under the isomorphism $\delta: K^{-1}(G / U) \rightarrow K^{0}(c(G / U), G / U),[q]$ is sent into $i^{*} \alpha\left(\rho, \rho^{\prime}\right)$ so $i^{*} \xi=[q]$. Let $\rho_{1}=\rho, \rho_{2}=\rho^{\prime}$ be the representations of $G$ on a vector space $V$.

Consider now

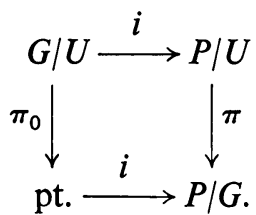

The vector bundles $\alpha\left(\rho_{j}\right)$ over $P / G$ can be written as $P \times_{\rho_{j}} V \rightarrow P / G$. Lifting to $P / U$ by $\pi^{*}, \pi^{*}\left(\alpha\left(\rho_{j}\right)\right)$ has as total space the set

$$
\left\{\left(p_{1} U, p_{2} \times_{\rho} v\right) \mid p_{1} G=p_{2} G ; p_{k} \in P, v \in V\right\} .
$$

A specific isomorphism $\phi_{j}$ of $P \times_{U} V$ with this vector bundle:

$$
P \times_{U} V \stackrel{\phi_{j}}{\longrightarrow} \pi^{*}\left(\alpha\left(\rho_{j}\right)\right)
$$

is given by

$$
\left(p \times_{U} v\right) \stackrel{\phi_{j}}{\longrightarrow}\left(p U, p \times_{\rho,} v\right)
$$

with inverse:

$$
\left(p_{1} \times_{U} \rho_{j}(g) v\right) \stackrel{\phi_{j}^{-1}}{\longleftarrow}\left(p_{1} U, p_{1} g \times_{\rho,} v\right) .
$$

We fix $p_{0}$ in $P$ and identify $i: G / U \rightarrow P / U$ with the map $g U \rightarrow p_{0} g U . \pi_{0}^{*} i^{*} \alpha\left(\rho_{j}\right)$ $=i^{*} \pi^{*} \alpha\left(\rho_{j}\right)$ has the total space

$$
\left\{\left(g_{1} U, g_{2} \times_{\rho} v\right)\right\}=(G / U) \times V
$$

since $G \times_{\rho,} V=V,\left(g \times_{\rho,} v\right)=\rho_{j}(g) v$, and $\phi_{j}^{-1}$ restricted to this space

$$
i^{*} \pi^{*} \alpha\left(\rho_{j}\right) \stackrel{\phi_{j}^{-1}}{\longrightarrow} G \times_{U} V
$$


is given by

$$
(g U, v) \stackrel{\phi_{j}^{-1}}{\longrightarrow}\left(g \times_{U} \rho_{j}(g)^{-1} v\right)
$$

and

$$
\left(g U, \rho_{j}(g) v\right) \stackrel{\phi_{j}}{\longleftarrow}\left(g \times_{U} v\right) .
$$

The clutching isomorphism $\pi^{*} \alpha\left(\rho_{2}\right) \rightarrow \pi^{*} \alpha\left(\rho_{1}\right)$ over $P / U$ is $\phi_{1} \phi_{2}^{-1}$. The restriction of this to $G / U$ is then

$$
\begin{gathered}
(G / U) \times V \stackrel{\phi_{2}^{-1}}{\longrightarrow} G \times_{U} V \stackrel{\phi_{1}}{\longrightarrow}(G / U) \times V, \\
(g U, v) \rightarrow\left(g \times_{U} \rho_{2}(g)^{-1} v\right) \rightarrow\left(g U, \rho_{1}(g) \rho_{2}(g)^{-1} v\right)=(g U, q(g U) v) .
\end{gathered}
$$

This concludes the proof of the proposition, and Theorem 4 is an immediate consequence.

\section{REFERENCES}

1. M. F. Atiyah, On the K-theory of compact Lie groups, Topology 4 (1965), 95-99.

2. R. Bott, Lectures on $K(X)$, Harvard Univ., Cambridge, Mass., 1962.

3. E. B. Dynkin, Maximal subgroups of the classical groups, Trudy Moskov. Mat. Obšč. 1 (1952), 39-166; Amer. Math. Soc. Transl. (2) 6 (1957), 245-378.

4. B. Harris, Suspensions and characteristic maps for symmetric spaces, Ann. of Math. (2) 76 (1962), 295-305.

5. N. Jacobson, Some groups of transformations defined by Jordan algebras. I, II, J. Reine Angew. Math. 201 (1959), 178-195; ibid. 204 (1960), 74-98.

6. P. G. Kumfel, On the homotopy groups of the exceptional Lie groups, Trans. Amer. Math. Soc. 120 (1965), 481-498.

7. J. Tits, Une classe d'algèbres de Lie en relation avec les algèbres de Jordan, Nederl. Akad. Wetensch. Proc. Ser. A 65 (1962), 530-535.

8. R. Bott, The space of loops on a Lie group, Michigan Math. J. 5 (1958), 35-61.

9. - The stable homotopy of the classical groups, Ann. of Math. (2) 70 (1959), 313-337.

10. L. Hodgkin, On the K-theory of Lie groups, Topology 6 (1967), 1-36.

11. S. Araki, On the Brouwer degrees of some maps of compact symmetric spaces, Topology 3 (1965), 281-290.

BROWN UNIVERSITY,

Providence, Rhode IsLAND 